EXTENDED REPORT

\title{
Does hydrotherapy improve strength and physical function in patients with osteoarthritis - a randomised controlled trial comparing a gym based and a hydrotherapy based strengthening programme
}

\author{
A Foley, J Halbert, T Hewitt, M Crotty
}

Ann Rheum Dis 2003;62:1162-1167. doi: 10.1136/ard.2002.005272

See end of article for authors' affiliations

.....................

Correspondence to: Professor M Crotty, Flinders University Department of Rehabilitation and Aged Care, C-Block, Repatriation General Hospital, Daws Road, Daw Park, South Australia 5041; maria.crotty@ rgh.sa.gov.au

Accepted 5 March 2003

\begin{abstract}
Objective: To compare the effects of a hydrotherapy resistance exercise programme with a gym based resistance exercise programme on strength and function in the treatment of osteoarthritis (OA).

Design: Single blind, three arm, randomised controlled trial.

Subjects: 105 community living participants aged 50 years and over with clinical OA of the hip or knee. Methods: Participants were randomised into one of three groups: hydrotherapy $(n=35)$, gym $(n=35)$, or control $(n=35)$. The two exercising groups had three exercise sessions a week for six weeks. At six weeks an independent physiotherapist unaware of the treatment allocation performed all outcome assessments (muscle strength dynamometry, six minute walk test, WOMAC OA Index, total drugs, SF-12 quality of life, Adelaide Activities Profile, and the Arthritis Self-Efficacy Scale).

Results: In the gym group both left and right quadriceps significantly increased in strength compared with the control group, and right quadriceps strength was also significantly better than in the hydrotherapy group. The hydrotherapy group increased left quadriceps strength only at follow up, and this was significantly different from the control group. The hydrotherapy group was significantly different from the control group for distance walked and the physical component of the SF-12. The gym group was significantly different from the control group for walk speed and self efficacy satisfaction. Compliance rates were similar for both exercise groups, with $84 \%$ of hydrotherapy and $75 \%$ of gym sessions attended. There were no differences in drug use between groups over the study period.

Conclusion: Functional gains were achieved with both exercise programmes compared with the control group.
\end{abstract}

O teoarthritis (OA) is the most common form of arthritis, affecting about $5-10 \%$ of the total Australian population. ${ }^{1}$ The development of $\mathrm{OA}$ is determined by age, genetic predisposition, previous injury, chronic stress across the joint, and abnormal mechanical forces caused primarily by obesity. ${ }^{12}$ Isolated quadriceps weakness has been associated with radiographic knee OA in older adults without a history of knee pain, and one descriptive study has demonstrated that decreased lower extremity strength was clearly associated with increased disability in people with OA. ${ }^{3}$ This suggests that muscle atrophy is either a consequence of, or a risk factor for, OA and is not simply due to patients avoiding loading painful osteoarthritic knee joints. ${ }^{4}$

Land based exercise regimens and strengthening exercise programmes have reported positive improvements in functional performance and reduction of the pain of $\mathrm{OA}^{5-9} \mathrm{~A}$ systematic review by Van Baar and colleagues evaluating the effectiveness of exercise therapy in patients with OA of the hip or knee ${ }^{10}$ concluded that exercise therapy is effective for this patient group as a range of beneficial effects were seen, including improved pain levels, physical function, and self reported disability. However, of the 11 randomised controlled trials identified, a wide variety of interventions were investigated, and overall, the quality of the studies was poor.

Hydrotherapy is often recommended to patients with arthritis as water provides a range of benefits, including the reduction of oedema, pain relief, and reduced loading on damaged arthritic joints. ${ }^{11}$ A systematic review of randomised controlled trials investigating hydrotherapy for the treatment of rheumatoid arthritis and $\mathrm{OA}^{12}$ found that all the studies had methodological flaws, with only three of the 10 studies reviewed scoring more than half of the maximum score available for methodological quality. Positive findings were detected in most of the trials, but the scientific evidence was considered weak and a conclusion about the efficacy of hydrotherapy for patients with arthritis could not be reached. Only three of the studies reviewed had studied patients with OA.

Methodological flaws of the studies appraised in the systematic reviews ${ }^{10} 12$ included lack of blinding of outcome assessors, and no intention to treat analysis.

Owing to the clinical importance of muscle strength in the development and progression of $\mathrm{OA}$, and the inconclusive evidence about the effectiveness of hydrotherapy for these patients, the current trial has directly compared standardised strengthening programmes in a hydrotherapy pool (water based) and gym (land based).

The direct comparison of these two forms of exercise is important in order to determine the efficacy of hydrotherapy, and further, to determine which method of exercise is more effective in strengthening the quadriceps muscles and improving functional performance in this patient group.

\section{METHODS}

The study was a randomised controlled trial, with blinded outcome assessment of 105 community living adults aged 50 and over with radiological diagnosis of OA of the hip or knee, or both. Eligible participants had to be able to independently read, write, and speak English, give informed consent, 
provide their own transport, and attend the Repatriation General Hospital three times a week for six consecutive weeks.

Participants were excluded if they had received physiotherapy or hydrotherapy in the past six weeks, were attending community exercise classes, had joint replacement surgery within the past 12 months or it was scheduled within the next 12 weeks, or if there was an indication of cognitive impairment. Participants were recruited from the physiotherapy, orthopaedic, and rheumatology departments of the Repatriation General Hospital (RGH), the orthopaedic department of the Flinders Medical Centre, and from the community through an advertisement.

Potential participants were sent information about the study, screened for eligibility, and invited to participate. A senior physiotherapist assessed the patients' capability of starting exercise in the gym or pool safely, and obtained written informed consent before baseline assessment. A computer generated randomisation list was created by a person external to the study and was managed by an external department. After baseline assessment, a person from the pharmacy department assigned participants to treatment group according to sequentially numbered, sealed, opaque envelopes, and the investigator was advised of group allocation by telephone. The intervention was started within five days of randomisation.

The exercise interventions consisted of either three water based, or three gym based exercise sessions a week for six weeks. Each 30 minute exercise session included a short warm up period, lower limb stretches, and a standardised set of resistance exercises with individually tailored progression of intensity. The control group received fortnightly telephone calls to record any changes in their condition, drug use, or injuries and were offered free exercise treatment at the RGH at the end of the study period.

All outcome measures were collected at baseline by a single trained investigator before randomisation, and at six weeks by a single "blinded" outcome assessor at the RGH. Clinical outcome measures included quadriceps strength and a six minute walk test. ${ }^{13}$ A series of self reported outcome questionnaires were also completed at baseline and at six weeks. The series of questionnaires included the Western Ontario and McMaster Universities (WOMAC) OA Index, ${ }^{14}$ consisting of three subscales including pain, stiffness, and physical function, with higher scores indicating greater disease severity. The Adelaide Activities Profile ${ }^{15}$ is categorised into four subscales, including domestic chores, household maintenance, social activities, and service to others, with higher scores indicating a higher level of daily activity. The Short Form-12 (SF-12) Health Survey ${ }^{16}$ measures quality of life and is divided into a physical and mental component, with higher scores indicating better physical and mental health. And the Arthritis Self-Efficacy Questionnaire ${ }^{17}$ consists of four subscales: pain, function, symptoms, and satisfaction, and higher scores here also indicate better health.

Data on demographics, medical history, and drugs were collected directly from the participants at baseline and used to describe the sample. Any injuries sustained during the study were recorded at six weeks and any changes in drug use were categorised as either; "change not related to OA", "increase in OA drug", or "decrease in OA drug".

The study was granted ethical approval by the RGH research and ethics committee.

\section{Quadriceps strength test protocol}

Isometric quadriceps strength was measured using a hydraulic leg extension machine modified to restrict leg extension at an angle of 110 degrees. Subjects were instructed to push the force gauge pad with their lower leg as hard as they could for five seconds. This was repeated three times on each leg with a 30 seconds rest between each effort, the final score being the mean of the three measures of peak force. Participants were familiarised with the test procedures and received vigorous verbal encouragement. Further details on the strength testing protocol are available from the first author upon request. Both intra- and intertester reliability for quadriceps strength were determined. The test-retest correlations for the baseline and follow up assessor were 0.758 and 0.897 , respectively and interrater reliability was also high: 0.761 .

\section{Exercise interventions}

The warm up in the water based exercise group consisted of walking forwards, sideways, and backwards through the water. The strengthening exercises included hip flexion and extension, hip adduction and abduction, knee flexion and extension, and knee cycling. One set of 10 repetitions was increased to three sets of 10 repetitions for each exercise, usually within the first week. Once three sets of 15 repetitions could be performed, weighted gaiters were fastened around the ankles to provide additional resistance. At this point, repetitions were dropped back to 10 and then increased to 15 as tolerated.

The warm up in the gym based exercise group involved about four minutes of stationary cycling. The strengthening exercises included seated bench press, hip adduction and abduction, knee extension, and double leg press. Participants started the programme working at either their 10 repetition maximum or just below their maximum, depending upon pain experienced during and after the initial exercise session. The same protocol for increasing exercise intensity in the water was also applied in the gym.

\section{Statistical analysis}

Statistical analysis was performed with SPSS version 10. An "intention to treat" approach was used in all analyses, and the last observation carried forward was used to impute data missing at follow up. Data are presented as medians and interquartile ranges, unless otherwise stated. An $\alpha$ level of 0.05 was used in statistical analyses.

Because most of the data followed non-normal distributions, non-parametric tests were used in all statistical analyses. The Kruskal-Wallis test was used to test for differences in outcome measures at baseline by treatment group, and the $\chi^{2}$ test was used to test for differences in baseline descriptors by treatment group. The effect of the intervention on the outcomes was determined using the Kruskal-Wallis test, and the Mann-Whitney test with a Bonferroni correction was used for subsequent post hoc pairwise comparisons. Wilcoxon signed ranks tests were used to determine within group changes from baseline to follow up.

To detect a change of $20 \%$ in the pain and physical functioning scales of the WOMAC OA Index between the two intervention groups, a sample size of 66 subjects was required ( 22 in each group). The sample size calculation was based on the assumption of an effect size of 1.0 with an $\alpha$ level of 0.05 and $90 \%$ power. To allow for drop outs and injuries, this sample was increased to 35 people in each group, a total of 105 participants.

\section{RESULTS}

Recruitment started on 1 April 2001 and was completed by the 25 March 2002 . Of the 429 people who were contacted to take part in the trial, 165 (38\%) were considered eligible. Of the eligible participants, $112(68 \%)$ consented, but it was considered unsafe for seven $(6 \%)$ to start an exercise programme. The remaining 105 participants were randomly 


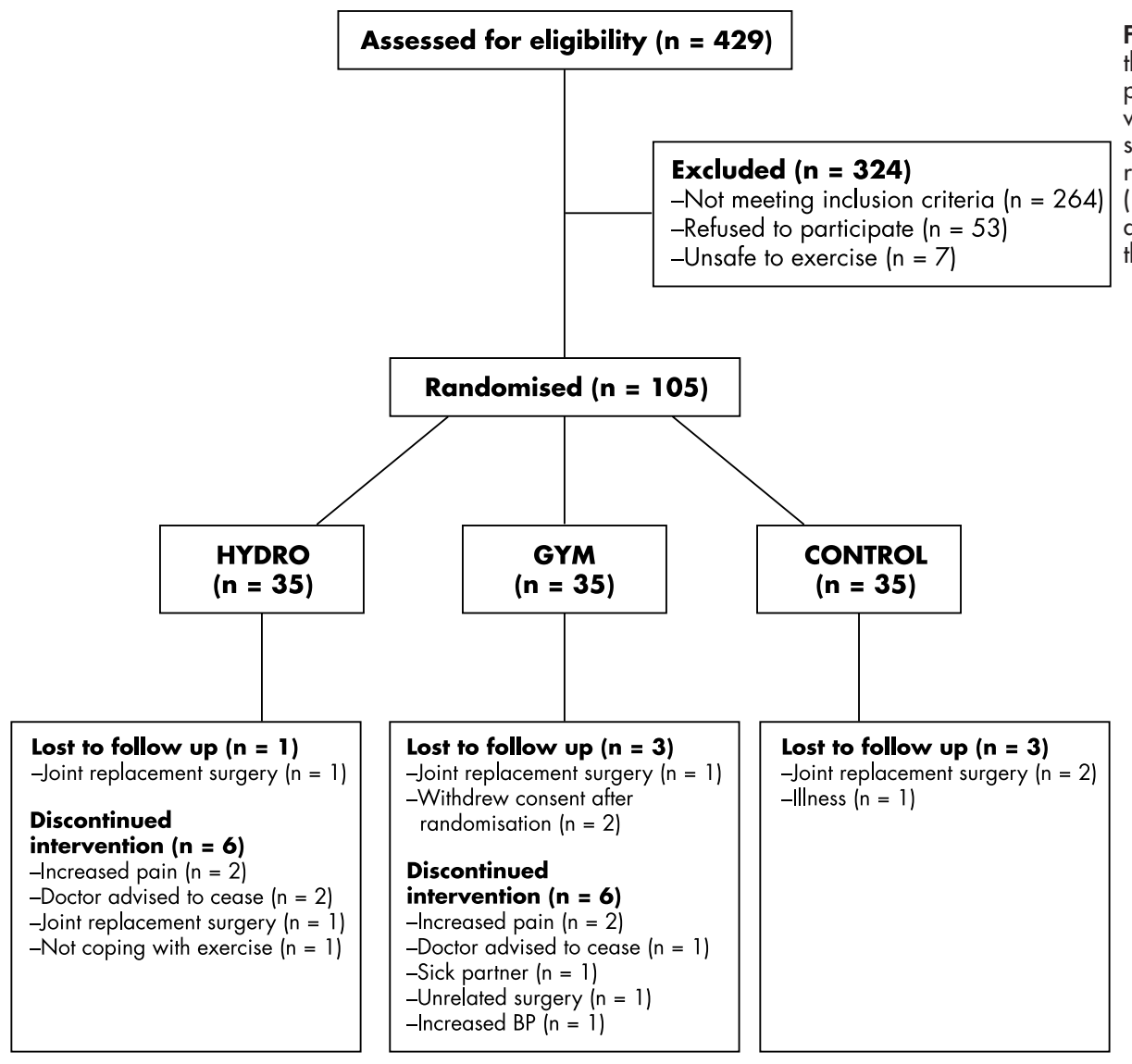

allocated to the hydrotherapy $(n=35)$, gym $(n=35)$, or control group ( $\mathrm{n}=35$ ) (fig $\mathrm{l}$ ).

Participants randomly allocated to the hydrotherapy and gym groups attended $84 \%$ and $75 \%$ of the exercise sessions, respectively. Six participants in each treatment group discontinued the intervention for a variety of reasons (fig 1). A small number of participants were lost to follow up in each group (fig 1).

Of the 105 participants, $52(49.5 \%)$ were women. The mean (SD) age of the sample was 70.9 (8.8) years. All participants in the study reported at least one comorbid condition other than OA. There was no significant difference between the groups for any of the baseline sample descriptors (table 1).

Table 1 also shows physical function, quadriceps strength, and self reported pain at baseline. There was no significant difference between the three groups at baseline for any of the clinical or self reported outcome measures, with the exception of self reported pain measured using the WOMAC OA Index. WOMAC pain scores were significantly higher in the hydrotherapy group than the gym group at baseline $(p=0.015)$. However there was no difference between groups for self reported pain using the Arthritis Self-Efficacy Scale.

Walking speed and distance improved significantly from baseline to follow up in both the hydrotherapy $(\mathrm{p}<0.001)$ and gym groups $(\mathrm{p}<0.001)$, but not in the control group. Pairwise comparisons of change scores showed that the hydrotherapy group was significantly better than the control group for distance walked and the gym group was significantly better than the control group for walking speed (fig 2).

The gym group significantly increased both left and right quadriceps strength from baseline to follow up $(p<0.001)$, and the hydrotherapy group significantly increased strength in the left quadriceps only $(\mathrm{p}=0.010)$. No change was seen in
Figure 1 Flow of study participants through selection and intervention protocols. Forty six (44\%) participants were recruited from orthopaedic surgery waiting lists, 16 (15\%) from rheumatology patient databases, 12 (11\%) from physiotherapy referrals, and $31(30 \%)$ from an advertisement in the local press (table 1). the control group for strength. Pairwise comparisons of change scores found that the gym group was significantly stronger than the control group for both left and right quadriceps strength, and significantly stronger than the hydrotherapy group for right quadriceps strength. The hydrotherapy group was significantly stronger than the control group for left quadriceps strength only (fig 2).

At follow up, the WOMAC pain score significantly declined from baseline in the hydrotherapy group, but the change in pain was not significantly different between groups (table 2). There were no significant changes from baseline or between groups for WOMAC function or stiffness. At follow up, no significant differences were found from baseline or between groups for self efficacy pain or function. The self efficacy satisfaction score significantly improved from baseline in the gym group, and there was a significantly greater improvement in satisfaction in the gym group compared with the control group (table 2). There were no significant differences from baseline scores, and there was also no significant difference between the groups for any of the Adelaide Activities subscales at follow up. At follow up, the hydrotherapy group showed a significant improvement from baseline in the SF-12 physical component score, and this was significantly different from the control group. The gym group showed a significant improvement from baseline in the SF-12 mental component score, but this change did not differ significantly between groups (table 2 ).

Significant differences in overall drug changes $(p=0.033)$ between the three groups were evident. However, the changes were such that increases and decreases in OA drug use largely cancelled each other out. The hydrotherapy group showed fewer changes in drug use, with only one participant increasing their OA drug. Four participants increased and five participants decreased their OA drug in the gym group, and 
Table 1 Descriptive data of the sample at baseline $(n=105)$.

\begin{tabular}{|c|c|c|c|c|}
\hline Baseline descriptor & Hydro $(n=35)$ & Gym $(n=35)$ & Control $(n=35)$ & $\begin{array}{l}\text { Total group } \\
(n=105)\end{array}$ \\
\hline \multicolumn{5}{|l|}{ Sex } \\
\hline Male & 20 & 15 & 15 & $53(50.5 \%)$ \\
\hline Female & 15 & 17 & 20 & $52(49.5 \%)$ \\
\hline \multicolumn{5}{|l|}{ Age (years) } \\
\hline Mean (SD) & $73.0(8.2)$ & $69.8(9.2)$ & $69.8(9.0)$ & $70.9(8.8)$ \\
\hline Range & $55-88$ & $51-88$ & $50-85$ & $50-88$ \\
\hline \multicolumn{5}{|l|}{ Recruited from: } \\
\hline Orthopaedic surgery waiting list & 16 & 12 & 18 & $46(44 \%)$ \\
\hline Community & 10 & 11 & 10 & $31(30 \%)$ \\
\hline Rheumatology database & 6 & 6 & 4 & $16(15 \%)$ \\
\hline Physiotherapy referral & 3 & 6 & 3 & $12(11 \%)$ \\
\hline \multicolumn{5}{|l|}{ Joints affected by OA } \\
\hline Hip joints (n) & 16 & 17 & 18 & $51(24 \%)$ \\
\hline Knee joints (n) & 43 & 46 & 37 & $126(60 \%)$ \\
\hline \multicolumn{5}{|l|}{ Comorbid conditions } \\
\hline Heart condition & 9 & 12 & 13 & $34(32 \%)$ \\
\hline Respiratory condition & 17 & 13 & 11 & $41(39 \%)$ \\
\hline Other & 33 & 33 & 34 & $100(95 \%)$ \\
\hline \multicolumn{5}{|l|}{ Pain* } \\
\hline WOMAC pain score (0-20) & $10.0(3.0) \dagger$ & $8.0(4.0)$ & $10.0(4.0)$ & $9.0(4.0)$ \\
\hline Arthritis Self-Efficacy Score (10-100) & $50.0(24.0)$ & $50.0(32.0)$ & $50.0(22.0)$ & $50.0(28.0)$ \\
\hline \multicolumn{5}{|l|}{ Function* ${ }^{*}$} \\
\hline Walking speed $(\mathrm{m} / \mathrm{s})$ & $0.99(0.49)$ & $0.97(0.39)$ & $1.11(0.46)$ & $1.02(0.40)$ \\
\hline Distance walked (m) & $257.3(282.8)$ & $336.2(240.1)$ & $388.1(320.9)$ & $336.0(296.0)$ \\
\hline \multicolumn{5}{|l|}{ Strength $(\mathrm{kg})^{*}$} \\
\hline Left quadriceps & $22.7(21.7)$ & $22.8(16.4)$ & $19.7(14.0)$ & $20.8(16.7)$ \\
\hline Right quadriceps & $22.5(24.9)$ & $19.7(9.9)$ & $22.4(17.3)$ & $20.8(18.3)$ \\
\hline
\end{tabular}

in the control group six participants increased and five decreased their OA drugs.

\section{DISCUSSION}

This study has shown that both the gym based and water based resistance exercise programmes successfully improved physical function. The gym based intervention was found to be more effective in improving muscle strength.

The significant increases in walking speed and distance found in the hydrotherapy group are supported by previous findings. ${ }^{18}{ }^{19}$ As far as we are aware, the effect of hydrotherapy on quadriceps strength has not been previously reported in this patient group. However, previous hydrotherapy studies have failed to show any significant changes in strength measured at the hip ${ }^{18}$ or in grip strength. ${ }^{2021}$ The increases in strength and function seen in the gym group are consistent with the findings of previous investigations of land based strengthening exercise programmes..$^{5-8}$

Improving a patient's ability to walk is important as it helps in maintaining independence to carry out activities of daily living, and the functional independence of older adults is also associated with decreased mortality and decreased admission into nursing homes and hospitals. ${ }^{22}$ Increased muscle strength around affected joints is a clinically important outcome as muscle strength provides increased shock absorbing capacity and improved joint stability to assist in the preservation of the diseased joint. ${ }^{23}$
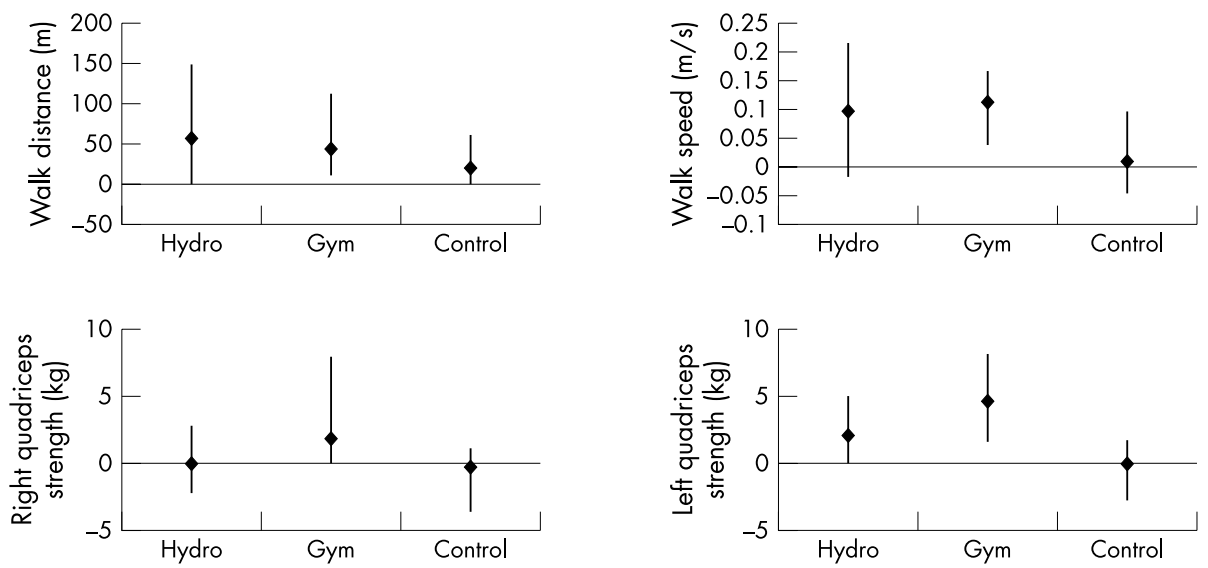

Figure 2 Medians and interquartile ranges for change scores in strength and physical function measures. For walking distance the hydro group was significantly different from the control group $(p=0.048)$. No other differences were significant. For walking speed the gym group was significantly different from the control group $(p=0.009)$. No other differences were significant. For right quadriceps strength the gym group was significantly different from the hydrotherapy $(p=0.030)$ and control groups $(p<0.001)$. No other differences were significant. For left quadriceps strength the gym group and the hydrotherapy groups were significantly different from the control group $(p<0.001, p=0.018$, respectively). No other differences were significant. 
Table 2 Self reported measures: WOMAC OA Index, Arthritis Self-Efficacy Scale, and SF-12 Health Survey

\begin{tabular}{|c|c|c|c|}
\hline Outcome & Baseline & Follow up & Median change \\
\hline \multicolumn{4}{|l|}{ WOMAC } \\
\hline \multicolumn{4}{|c|}{ Function score (0-68) } \\
\hline Hydro & $34.0(16.0)$ & $33.0(17.0)$ & $-1.0(10.0)$ \\
\hline Gym & $28.0(13.0)$ & $27.0(12.0)$ & $-1.0(11.0)$ \\
\hline Control & $37.0(17.0)$ & $37.0(13.0)$ & $0.0(8.0)$ \\
\hline \multicolumn{4}{|c|}{ Stiffness score (0-8) } \\
\hline Hydro & $5.0(2.0)$ & $4.0(3.0)$ & $0.0(1.0)$ \\
\hline Gym & $4.0(2.0)$ & $4.0(2.0)$ & $0.0(2.0)$ \\
\hline Control & $4.0(3.0)$ & $4.0(3.0)$ & $0.0(2.0)$ \\
\hline \multicolumn{4}{|c|}{ Pain score (0-20) } \\
\hline Hydro & $10.0(3.0)$ & $10.0(4.0)^{*}$ & $-1.0(3.0)$ \\
\hline Gym & $8.0(4.0)$ & $8.0(5.0)$ & $0.0(3.0)$ \\
\hline Control & $10.0(4.0)$ & $10.0(4.0)$ & $1.0(3.0)$ \\
\hline \multicolumn{4}{|c|}{ Self efficacy } \\
\hline \multicolumn{4}{|c|}{$\begin{array}{l}\text { Symptoms score } \\
(10-100)\end{array}$} \\
\hline Hydro & $65.0(23.3)$ & $63.3(26.7)$ & $-1.7(25.0)$ \\
\hline Gym & $70.0(26.7)$ & $70.0(30.0)$ & $5.0(15.0)$ \\
\hline Control & $63.3(31.7)$ & $58.3(28.3)$ & $-1.7(11.7)$ \\
\hline \multicolumn{4}{|c|}{$\begin{array}{l}\text { Satisfaction score } \\
(10-100)\end{array}$} \\
\hline Hydro & $50.0(20.0)$ & $50.0(25.0)$ & $0.0(20.0)$ \\
\hline Gym & $50.0(25.0)$ & $60.0(30.0) \dagger$ & $5.0(15.0)$ \\
\hline Control & $55.0(30.0)$ & $50.0(40.0)$ & $0.0(15.0)$ \\
\hline \multicolumn{4}{|l|}{ SF-12 } \\
\hline \multicolumn{4}{|c|}{ Physical score $(16-57)$} \\
\hline Hydro & $31.4(7.9)$ & $37.1(12.7) \ddagger^{* *}$ & $2.9(7.7)$ \\
\hline Gym & $30.7(11.2)$ & $31.4(12.7)$ & $2.8(10.6)$ \\
\hline Control & $30.9(11.2)$ & $28.8(11.0)$ & $-0.1(8.1)$ \\
\hline \multicolumn{4}{|c|}{ Mental score (25-70) } \\
\hline Hydro & $53.4(15.7)$ & $53.3(15.5)$ & $0.0(10.0)$ \\
\hline Gym & $51.8(21.2)$ & $57.9(19.5) \S$ & $2.2(7.3)$ \\
\hline Control & $50.5(16.9)$ & $50.5(14.0)$ & $0.0(8.8)$ \\
\hline \multicolumn{4}{|c|}{ 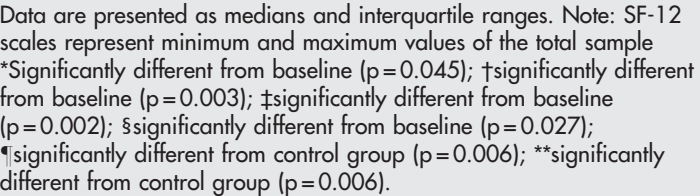 } \\
\hline
\end{tabular}

Our study failed to find any significant difference between the two intervention groups for improvements in walking speed and distance. Previous studies that have investigated hydrotherapy versus home based exercise, ${ }^{18}$ land based resistance exercise, ${ }^{19}$ and electrotherapy and exercise, ${ }^{24}$ have also found no significant difference between intervention groups for walking ability measured by the time taken to walk a fixed distance ${ }^{18} 19$ and gait assessment. ${ }^{24}$

The hydrotherapy group reported significant improvement in the physical component score of the SF-12 quality of life measure; in contrast, the gym group reported significant improvement in the mental component score. Self reported improvement was also reported in the gym group for two scales of the arthritis self efficacy questionnaire, indicating increased satisfaction with their ability to manage their arthritic pain and other arthritis symptoms.

For other self reported outcomes, we suggest that lack of change may be due to participants overestimating their capabilities at baseline by assuming that they can do more than they actually can, and after six weeks of exercise therapy they have a better idea of their true physical capabilities and provide a more accurate reflection of this at follow up. However, it is likely that owing to the heterogeneity of the sample and the smaller than expected effect size, we were underpowered to detect a significant change in these self reported measures.

The intensity, volume, and frequency of the exercise interventions was much higher than the American Geriatrics Society recommendations for strength training in patients with $\mathrm{OA}^{25}$ and the hydrotherapy programmes normally prescribed within the RGH. Higher intensity exercise was implemented in the current study because previous strength training studies of patients with OA have recorded no adverse effects or exacerbation of symptoms, and exercise has not been shown to accelerate disease progression. ${ }^{25}{ }^{26}$ We also found that the exercise interventions did not increase self reported OA pain or symptom scores.

Over $40 \%$ of the sample were recruited from orthopaedic surgery waiting lists, indicating that a large percentage of the sample had moderate to severe OA requiring a hip or knee joint replacement. Therefore, the results of the current study support the prescription of higher intensity exercise for patients, even for those with severe OA who are awaiting a joint replacement. This finding is consistent with earlier work that showed that an exercise programme could be successfully administered in patients with OA severe enough to warrant referral to an orthopaedic surgeon. ${ }^{8}$

It was our intention to match the exercise intensity between the two intervention groups as closely as possible. However, progressive overloading of the musculature and loading through the eccentric phase of muscle contraction is not possible in water as it is on land. Therefore, owing to the nature of hydrotherapy, the exercise intensity would not have been as high in the water based group, hence the greater increases in strength seen in the gym group. However, the hydrotherapy programme had an underlying aerobic training component, as higher and faster repetitions were used to increase the exercise intensity. Furthermore, the hydrotherapy group continuously worked for the full half hour session, moving immediately from one exercise to the next. Owing to these characteristics of the hydrotherapy programme, it is expected that the participant's heart rate (although not measured) was raised and maintained at a higher level for the duration of each exercise session. Patients with OA typically have low cardiovascular fitness, ${ }^{26}$ therefore it is expected that the hydrotherapy programme possibly produced increases in aerobic capacity, explaining the significant increase in physical function without the same increases in strength as observed in the gym group.

One potential limitation of the study is how truly representative the sample is of the OA population in general. Firstly, most subjects entering the trial were keen to take part in the exercise intervention in the hope that they would benefit from the exercise. However, not all patients with OA would be as keen to exercise as regularly or as intensively as the subjects in the current study. Although the recruitment of subjects was based on the presence of hip or knee OA and not fitness, it is believed that research projects involving physical activity will generally attract people who are interested in exercise. ${ }^{27}$ Secondly, only participants who could provide their own transport to the RGH three times a week were eligible to take part in the trial.

Lastly, changes in drug use were not controlled over the study period. However, documentation of drugs at baseline and follow up showed that participation in land based exercise, water based exercise, or no exercise resulted in no net increase or decrease in drug use. This suggests that the results are not likely to have been influenced by changes in drug use as the changes that occurred largely cancelled each other out in each group.

In summary, this study has shown that both the gym and hydrotherapy interventions produce positive functional outcomes for patients with OA. However, it seems that hydrotherapy may be more suitable for aerobic based exercise programmes and the gym based exercise more suitable for strengthening programmes. Hydrotherapy provides the optimal environment for patients with OA to exercise aerobically, and at higher intensities than would be possible on land, 
owing to the reduction of joint loading. ${ }^{11}$ Patients with severe OA who find it painful to weight bear for extended periods may find that water provides the appropriate environment in which they can exercise at intensities that may confer significant health benefits. However, to improve joint stability and shock absorbing capacity through improved muscle strength, land based resistance exercise should be recommended as loading during eccentric muscle contractions and progressive resistance can be more effectively applied on land.

Currently published reports indicate that few high quality trials have been carried out to examine the use of hydrotherapy in the treatment of patients with OA. Further research into hydrotherapy should investigate the long term effects of this form of exercise in patients with OA, and should also aim at measuring the effect of hydrotherapy on cardiovascular fitness.

\section{ACKNOWLEDGEMENTS}

The authors acknowledge Professor J Krishnan and staff of the orthopaedic department (FMC/RGH); Associate Professors M Ahern and M Smith and staff of the rheumatology department (RGH) for forwarding patients for subject recruitment; Jennifer Robertson for conducting the outcome assessments; Kylie Lange for assistance with statistical analysis; Lynne Giles for assistance with the preparation and review of the manuscript, and all the participants who took part in the study, as without their participation, the research would not have been possible.

\section{Authors' affiliations}

A Foley, J Halbert, M Crotty, Flinders University Department of Rehabilitation and Aged Care, Repatriation General Hospital, South Australia

T Hewitt, Physiotherapy Department, Repatriation General Hospital, South Australia

This project was undertaken as part of the first author's requirements for the award of BSc(Hons) in 2001 at the Flinders University of South Australia.

\section{REFERENCES}

1 The Arthritis Foundation of Australia. The prevalence, cost and disease burden of arthritis in Australia. Canberra, ACT: Access Economics Pty Ltd 2001:1-2.

2 Kerr RG, Al-Kawan RH. Osteoarthritis: a primary care approach for physicians in 2000 and beyond. Saudi Med J 2001;22:403-6.

3 McAlindon TE, Cooper C, Kirwan JR, Dieppe PA. Determinants of disability in osteoarthritis of the knee. Ann Rheum Dis 1993:52:258-62

4 Slemenda C, Brandt KD, Heilman DK, Mazzuca S, Braunstein EM, Katz BP et al. Quadriceps weakness and osteoarthritis of the knee. Ann Intern Med 1997; 127:97-104.
5 Fransen M, Crosbie J, Edmonds J. Physical therapy is effective for patients with osteoarthritis of the knee: a randomised controlled clinical trial. J Rheumatol $2001 ; 28: 156-64$

6 Deyle GD, Henderson NE, Matekel RL, Ryder MG, Garber MB, Allison SC. Effectiveness of manual physical therapy and exercise in osteoarthritis of the knee. A randomised controlled trial. Ann Intern Med 2000;132:173-81.

7 Hurley MV, Scott DL. Improvements in quadriceps sensorimotor function and disability of patients with knee osteoarthritis following a clinically practicable exercise regime. $\mathrm{Br} J$ Rheumatol 1998;37:1 181-7.

8 Rogind H, Bibow-Nielsen B, Jensen B, Moller HC, Frimodt-Moller H, Bliddal H. The effects of a physical training programme on patients with osteoarthritis of the knees. Arch Phys Med Rehabil 1998;79:1421-7.

9 Maurer BT, Stern AG, Kinossian B, Cook KD, Schumacher HR Jr. Osteoarthritis of the knee: isokinetic quadriceps exercise versus an educational intervention. Arch Phys Med Rehabil 1999;80:1293-9.

10 Van Baar ME, Assendelft WJJ, Dekker J, Oostendorp RAB, Biilsma JWJ. Effectiveness of exercise therapy in patients with osteoarthritis of the hip or knee. A systematic review of randomised controlled trials. Arthritis Rheum 1999;42:1361-9.

11 Simon L, Blotman F. Exercise therapy and hydrotherapy in the treatment of the rheumatic diseases. Clin Rheum Dis 1981;7:337-47.

12 Verhagen AP, de Vet HCW, de Bie RA, Kessels AGH, Boers M, Knipschild PG. Balneotherapy for rheumatoid arthritis and osteoarthritis. Cochrane Database Syst Rev, $2001 ;(1)$.

13 Butland RJA, Pang J, Gross ER, Woodcock AA, Geddes DM. Two-, six-, and 12 minute walking tests in respiratory disease. BMJ 1982;284:1607-8.

14 Bellamy N. Osteoarthritis - an evaluative index for clinical trials. Hamilton, Canada: McMaster University, 1982 (MSc thesis.).

15 Clark MS, Bond MJ. The Adelaide Activities Profile: A measure of the lifestyle activities of elderly people. Aging Clin Exp Res 1995;7:174-84.

16 Jenkinson C, Layte R. Development and testing of the UK SF-12 (short form health survey). J Health Serv Res Policy 1997;2:14-18.

17 Lorig K, Chastain RL, Ung E, Shoor S, Holman HR. Development and evaluation of a scale to measure perceived self efficacy in people with arthritis. Arthritis Rheum 1989;32:37-44.

18 Green J. Home exercises are as effective as outpatient hydrotherapy for osteoarthritis of the hip. Br J Rheumatol 1993;32:812-15.

19 Wyatt FB, Milam S, Manske RC, Deere R. The effects of aquatic and traditional exercise programmes on persons with knee osteoarthritis. J Strength Cond Res 2001;15:337-40

20 Alexander MJL, Butcher JE, MacDonald PB. Effect of a water exercise programme on walking gait, flexibility, strength, self reported disability and other psycho-social measures of older individuals with arthritis. Physio Canada 2001;53:203-11.

21 Minor MA, Hewett JE, Webel RR, Anderson SK, Kay DR. Efficacy of physical conditioning exercise in patients with rheumatoid arthritis and osteoarthritis. Arthritis Rheum 1989;32:1396-1405.

22 Sharkey NA, Williams NI, Guerin JB. The role of exercise in the prevention and treatment of osteoporosis and osteoarthritis. Nurs Clin North Am 2000;35:209-21.

23 Hurley MV. The role of muscle weakness in the pathogenesis of osteoarthritis. Rheum Dis Clin North Am 1999:25:283-98.

24 Sylvester KL. Investigation of the effect of hydrotherapy in the treatment of osteoarthritic hips. Clin Rehabil 1989;4:223-8.

25 American Geriatrics Society. Exercise prescription for older adults with osteoarthritis pain: consensus practice recommendations. A supplement to the AGS Clinical Practice Guidelines on the management of chronic pain in older adults. J Am Geriatr Soc 2001;49:808-23.

26 Ettinger WH Jr, Burns R, Messier SP, Applegate W, Rejeski WJ , Morgan T, et al. A randomised trial comparing aerobic exercise and resistance exercise with a health education programme in older adults with knee osteoarthritis: The Fitness Arthritis and Seniors Trial (FAST). JAMA 1997;227:25-31.

27 Greig CA, Young A, Skelton DA. Exercise studies with elderly volunteers. Age Ageing 1994;23:185-9. 